\title{
Activity of Some Medicinal Plants Against Phytopathogenic Fungi
}

\author{
S. Sivanandhan ${ }^{1}$, P. Ganesan ${ }^{1,2}$, A. Jackson ${ }^{1}$, S. Darvin ${ }^{3}$, M.G.Paulraj ${ }^{2}$, S. Ignacimuthu ${ }^{4}$ * \\ ${ }^{1}$ Division of Microbiology, Entomology Research Institute, Loyola College, Nungambakkam, Chennai, India \\ ${ }^{2}$ Divition of Vector control, Entomology Research Institute, Loyola College, Nungambakkam, Chennai, India \\ ${ }^{3}$ Division of Ethanopharmacology, Entomology Research Institute, Loyola College, Nungambakkam, Chennai, India \\ ${ }^{4}$ Emeritus Scientist, Entomology Research Institute, Loyola College, Nungambakkam, Chennai, India \\ * Corresponding author: eriloyola@hotmail.com; Tel. +914428178348
}

Available online at: www.isroset.org

Received: 05/Oct/2018, Accepted: 22/Oct/2018, Online: 31/Oct/2018

\begin{abstract}
Crude extracts of 17 medicinal plants were examined for their antifungal properties against phytopathogenic fungi. Hexane, chloroform and methanol extracts were tested using disc diffusion method at concentrations varying from 1.25 to 5 $\mathrm{mg} /$ disc. Among these, hexane extract of Blumea mollis was the most effective against Aspergillus flavus with zone of inhibition of $23 \mathrm{~mm}$ at $5 \mathrm{mg} /$ disc concentration. The Minimal inhibitory concentration (MIC) of B. mollis hexane extract against A. flavus, A. niger and Botrytis cinerea were $0.623,0.825$ and $0.622 \mathrm{mg} / \mathrm{ml}$ respectively. Terpenoids, saponins, anthraquinones and anthocyanins were detected in the hexane extract of B. mollis. Subsequently, GC-MS analysis of the extract revealed the main constituent to be cycloheptane (34.21\%). This could have potential use as a fungicide against phytopathogens.
\end{abstract}

Keywords: Phytopathogenic fungi, plant extract, disc diffusion, MIC, GCMS

\section{INTRODUCTION}

Crop production faces many problems among which fungal diseases are major cause of yield loss throughout the world resulting in excessive economic burdens [1]. Yield losses caused by pathogens, animals and weeds altogether range between 20 and $40 \%$ in global agricultural productivity [2]. Though majority of the plant diseases are caused by phytopathogenic fungi, some diseases are also caused by bacteria, nematodes and viruses [3]. Fungi are known to infect the plants during the developmental stages and also at post-harvest period [4]. Degradation of grains, vegetables and fruits caused by pathogenic fungi may lead to loss of entire product. Involvement of Aspergillus sp. along with variety of other fungi like Botrytis cinerea and Fusarium sp.) in the accumulation of fungal toxins in seed and loss of nutritional quality has been reported [5]. Synthetic chemical agents are heavily used to control the phytopathogenic fungi, which results in resistance development in the pathogens and accumulation of chemicals in the environment [6,7]. Synthetic chemicals are also toxic to human beings and cause carcinogenicity and teratogenicity [8]. Numerous countries in the yester years have proscribed the agricultural pesticides due to their toxicity to non-targets such as humans [9]. So there is a need for plant-based compounds for ecofriendly applications to control the crop damage caused by fungi, bacteria, nematodes and other organisms. Plants produce secondary metabolites such as flavonoids, alkaloids, teripinoids etc., for aforementioned reason as well as other uses as antimicrobials, insecticides, nematicides along with other activities $[10,11,12,13,14,15,16]$. Many medicinal plants have fungicidal properties [17] against different fungal species including phytopathogenic fungi. Blumea mollis is an aromatic annual herb. It is a weed commonly found in India, Sri Lanka and Myanmar [18]. The leaf has been traditionally used for skin diseases and the boiled herb has been used to treat diarrhea [19]. The whole plant mixed with garlic is used to treat leucorrhoea [20]. The present study was carried out to investigate the inhibitory effects of different organic solvent extracts of 17 medicinal plants against 11 phytopathogenic fungi and minimal inhibitory concentration was found out for the most effective extract. 


\section{MATERIALS AND METHODS}

Plant collection and identification

Seventeen medicinal plants were collected from different places in the state of Tamil Nadu, India (Table 1). All the plant species were collected during flowering stage in the month of March to May 2016. The taxonomical identification of the plant species was performed by taxonomist Dr. Pandikumar, Entomology Research Institute (ERI), Loyola College, Chennai. Voucher specimens were deposited in the herbarium at ERI.

Preparation of crude extract

The plant materials were shade-dried at room temperature and powdered using electric blender. The powdered plant materials $(100 \mathrm{~g})$ were sequentially extracted with hexane, chloroform and methanol. After $72 \mathrm{~h}$ of soaking of the plant material in each solvent, the extract was filtered through Whatman filter paper using vacuum. Solvent in the extract was removed using rotary vacuum evaporator at $45-55^{\circ} \mathrm{C}$ and the crude extracts were stored at $4^{\circ} \mathrm{C}$ in the refrigerator for further bioassay.

Fungal culture and spore collection.

Eleven phytopathogens namely Alternaria alternata, Aspergillus flavus, Aspergillus niger, B. cinerea, Bipolaris oryzae, Curvularia lunata, Curvularia oryzae, Fusarium solani, Fusarium oxysporum, Macrophomina sp and Trichoderma sp were collected from the Department of Plant Pathology, Annamalai University, Tamil Nadu, India. All the fungal cultures were grown in Potato dextrose agar medium for 7-10 days and spore suspension was filtered with sterile muslin cloths; conidia spores were collected and spore suspension was adjusted to $2-10^{5}$ spores $/ \mathrm{ml}$ using haemocytometer [21].

Antifungal susceptibility test

Antifungal activity of 17 plants was screened by following the kirby bauer disk diffusion method [22]. Sterile Potato Dextrose Agar $(20 \mathrm{ml})$ (PDA, HiMedia) was taken in petri plates and the agar was allowed to solidify for 5 minutes; the fungal spore suspension was swabbed uniformly on the agar. Different concentrations of plant extracts viz., $1.25,2.5$ and $5 \mathrm{mg} / \mathrm{disc}$ were used [23] for diffusion method and the reference control, Hexaconazole $(5 \mathrm{mg} / \mathrm{ml})$ was used as positive control for comparison. The extract was dissolved in water $+2 \%$ Dimethyl sulfoxide (DMSO) and was loaded on separate sterile discs $(6 \mathrm{~mm}$ diameter). The extract-loaded discs were placed on the surface of agar medium and the extract was allowed to diffuse for 5 minutes. The plates were kept in incubator at $27^{\circ} \mathrm{C}$ for 48 to $96 \mathrm{~h}$. At the end of incubation, inhibition zones formed around the sterile disc were measured with a Vernier caliper in millimeter (Antibiotic zone scale). This study was performed in triplicate.

Minimal inhibitory concentration

Minimum Inhibitory Concentration (MIC) was found out using microdilution technique using standard method (CLSI, 1998) (23). The extracts were dissolved in water $+2 \%$ Dimethyl Sulfoxide (DMSO). The initial test concentration of the extract was $1 \mathrm{mg} / \mathrm{ml}$. Hexaconazole and carbendazim wereused as standard drugs. Test contration was serially diluted (5 mg to 0.1$)$ in a 96 well plate. Each well was inoculated with $10 \mu \mathrm{l}$ of spore suspension containing 104 spores $/ \mathrm{ml}$ of fungi. The plates were incubated at $27^{\circ}$ for 3 to 5 days. MIC was performed as the lowest extract concentration, showing no visible fungal growth after incubation time. $10 \mu \mathrm{l}$ of tested broth was placed on the sterile PDB medium for fungi and incubated at respective temperature. The MIC for fungi was determined as the lowest concentration of the extract inhibiting the visual growth of the test fungal cultures on the 96 well plate.

Preliminary phytochemical analysis of B. mollis extract

Qualitative phytochemical analysis of the active plant extract was done following the method of Yadav et al. (2014) [24].

The GCMS analysis

The GCMS analysis of hexane extract of $B$. mollis leaves was done using Agilent Technologies GC systems with model name GC-7890A/MS-5975C (Agilent Tec hnologies, Santa Clara, CA, USA) equipped with HP-5MS column $30 \mathrm{~m}$ in length $\times$ $0.25 \mathrm{~mm}$ in diameter $\times 0.25 \mu \mathrm{m}$ in thickness film. Spectroscopic finding by GCMS involved an electron ionization system which consumed high energy electrons $(-70 \mathrm{eV})$. The carrier gas of pure helium gas $(99.995 \%)$ was used with flow rate of 1.0 $\mathrm{ml}$ per min. The oven program as follows initial temperature was set at $50{ }^{\circ} \mathrm{C}$, held at $1 \mathrm{~min}$; the second step was at $170{ }^{\circ} \mathrm{C}$ for $0 \mathrm{~min}$ and the final step was at $300{ }^{\circ} \mathrm{C}$ for $10 \mathrm{~min} .2 \mu \mathrm{l}$ of the prepared extract $1 \%$ diluted with respective solvent was injected in a splitless mode with average velocity of $36.445 \mathrm{~cm} / \mathrm{sec}$. Comparative quantity of the chemical compounds present in hexane extract of $B$. mollis was expressed as percentage based on peak area formed in the chromatogram. 


\section{RESULTS AND DISCUSSION}

Table 1 shows the details of botanical name, family, local name, collection time and yield of crude extracts. The result of the antifungal screening of the crude extracts of all plant species is shown in table 2. Among the 17 plants screened, the $B$. mollis hexane extract showed promising activity against tested fungi. The hexane extract of $B$. mollis showed (Figure 1) maximum zones of inhibition against A. flavus $(23 \mathrm{~mm})$, A.niger $(18.33 \mathrm{~mm})$, B. cinerea $(18.33 \mathrm{~mm})$, C.oryzae $(16.33 \mathrm{~mm})$, C. lunata $(20.66 \mathrm{~mm}), F$. solani $(17.33 \mathrm{~mm})$ and $F$. oxysporum $(14.33 \mathrm{~mm})$. The chloroform extract showed moderate activity against $F$. solani, B. cinerea and C. oryzae (Table 3). The MIC of B. mollis hexane extract was low against A. flavus $(0.623 \mathrm{mg} / \mathrm{ml}), A$. niger $(0.825 \mathrm{mg} / \mathrm{ml})$, B. cinerea $(0.622 \mathrm{mg} / \mathrm{ml})$, C. oryzea. $(1.25 \mathrm{mg} / \mathrm{ml})$, C. lunata $(0.821 \mathrm{mg} / \mathrm{ml})$ and $F$. solani $(1.15 \mathrm{mg} / \mathrm{ml})$ (Table 4).

The commercial drug Hexaconazole showed MIC values of against A. flavus, B. cinerea $1.25 \pm 1 \mathrm{mg} / \mathrm{ml}$, A. niger $(0.622$ $\mathrm{mg} / \mathrm{ml}$ ) and $C$. oryzea, $C$. lunata, $F$. solani $F$. oxysporum did not show any activity, even at $5 \mathrm{mg} / \mathrm{ml}$ concentration. Fungicide Bevistine showed MIC value of $(1.25 \pm 0 \mathrm{mg} / \mathrm{ml})$ against $A$. flavus, B. cinerea, C. oryzea followed by A. niger, C. lunata, $F$. oxysporum $(0.622 \mathrm{mg} / \mathrm{ml})$ and $F$. solani $(0.312 \pm 0 \mathrm{mg} / \mathrm{ml})$. The chemical constituents were identified using GCMS (Figure 2). The results showed that 27 compounds were detected (Table 6) in the hexane extract of $B$. mollis among which three compounds namely Bicyclo[7.2.0]undec-4-ene, 4,11,11-trimethyl-8-methylene-,[1R-(1R*,4Z,9S*)]- (18.24\%), Cycloheptane, 4-methylene-1-methyl-2-(2-methyl-1-propen-1-yl)-1-vinyl(34.21\%) and Caryophyllene oxide (26.73\%) were found as major components.

Fungal diseases cause severe growth reduction and yield loss in many agricultural, ornamental and horticultural crops. Fungal spores easily spread through air, water and soil and infect other plants. At present, ecofriendly biochemicals are more preferred to control plant pathogens to avoid environmental contamination, non-target effects and side effects in humans. Medicinal plant extracts are promising sources of biologically active chemicals that may be used to control phytopathogenic fungi [25, 26]. In the present study, 51 crude extracts from 17 medicinal plants were screened against important phytopathogenic fungi. B. mollis hexane extract showed the most potential activity against many pathogenic fungi. B. mollis is a weed; but has many medicinal properties. Native people of in Andhra Pradesh in India are using B. mollis to treat hepatotoxicity, asthma and dropsy [18]. The methanol extract of $B$. mollis had hepatoprotective activity in wistar rats [27]. The water extract $B$. mollis showed anti-inflammatory, antioxidant activity and anticancer activities [28].

B. mollis possessed antifungal activity against 11 phytopathogens. All other plants, tested in this study did not show antifungal activity against the tested pathogenic fungi. Hexane extract presented maximum zone of inhibition of $23 \mathrm{~mm}$ against $A$. flavus. Hexane extract of $B$. mollis used in the present study was found to be the better antifungal agent than many previously reported plant extracts. For example Chandrasekaran and Venkatesalu [29] tested the methanol and aqueous extracts of Syzygium jambolanum seed against 9 fungal pathogens at $1 \mathrm{mg} / \mathrm{ml}$ concentration. The extract presented the highest zone of inhibition of $15 \mathrm{~mm}$ against A. flavus and A. fumigatus. Ethanol and aqueous extracts of Acalypha wilkesiana showed maximum zone of inhibition of 10 and $15 \mathrm{~mm}$ respectively at $30 \mathrm{mg} / \mathrm{ml}$ dose against A. flavus [30]. Ethanol extract of Nymphaea nouchali seeds presented $10 \mathrm{~mm}, 10 \mathrm{~mm}$ and $11 \mathrm{~mm}$ zones of inhibitions at $1 \mathrm{mg} / \mathrm{ml}$ against A. niger, Penicllium $\mathrm{sp}$, and Curvalaria sp. respectively [31]. Similarly, the methanol extract of the bark and leaves of Acacia nilotica showed $12 \mathrm{~mm}$ zone of inhibition against A. flavus and leaf extract of Zizphus mauritian recorded $11 \mathrm{~mm}$ zone of inhibition at $10 \mathrm{mg} / \mathrm{ml}$ [32]. In our study, the MIC value of hexane extract of $B$. mollis was recorded as $0.625 \mathrm{mg} / \mathrm{ml}$, which clearly showed its potential antifungal activity against $A$. flavus, A. niger and B. cinearae. Similar study by Breda et al. (2016) [4] reported that the ethanol extracts of Correio brasiliense (pequi) leaves and fruit peel were active against Alternaria alternata, Alternaria solani and Venturia pirina with MIC ranging between 350 and $1000 \mu \mathrm{g} / \mathrm{mL}$. In another study, the root part of Hypochaeris radicata presented MIC values of 200 and $600 \mu \mathrm{g} / \mathrm{mL}$ against A. niger and Mucor sp. respectively [33]. The ethanol extract of Rhus muelleri showed growth inhibition against $F$. oxysporum at MIC value of 11,793 ppm [34], which was very high compared to the MIC value of $B$. mollis hexane extract in the present study.

The GCMS study showed that hexane extract of B. mollis had three major compounds namely Bicyclo[7.2.0]undec-4-ene, 4,11,11-trimethyl-8-methylene-,[1R-(1R*,4Z,9S*)]- (18.24\%), Cycloheptane, 4-methylene-1-methyl-2-(2-methyl-1-propen-1yl)-1-vinyl(34.21\%) and Caryophyllene oxide (26.73\%). Previously Senthilkumar et al. (2008) [35] reported that essential oil of B. mollis contained linalool (19.43\%) and $\gamma$-elemene (12.19\%) as major ingredients. In a previous study caryophyllene oxide has been reported as an antifungal agent (36).

\section{CONCLUSION AND FUTURE SCOPE}

In the present $B$. mollis hexane extract showed promising activity against major phytopathogenic fungi at very low concentration. Since $B$. mollis is already used as medicinal plant, it can be included in the control of plant pathogenic fungi. $B$. mollis is a weed and easily available one. Secondary metabolites of plant origins have noticeable impressions on many research 
fields and added benefit of producing inexpensively. Finding of more such products is a boon for eco-friendly crop management, in turn they ameliorate the redundancy of synthetic chemicals. Furthermore extensive research is required to identify much more bioagents antagonistic to phytopathogens.
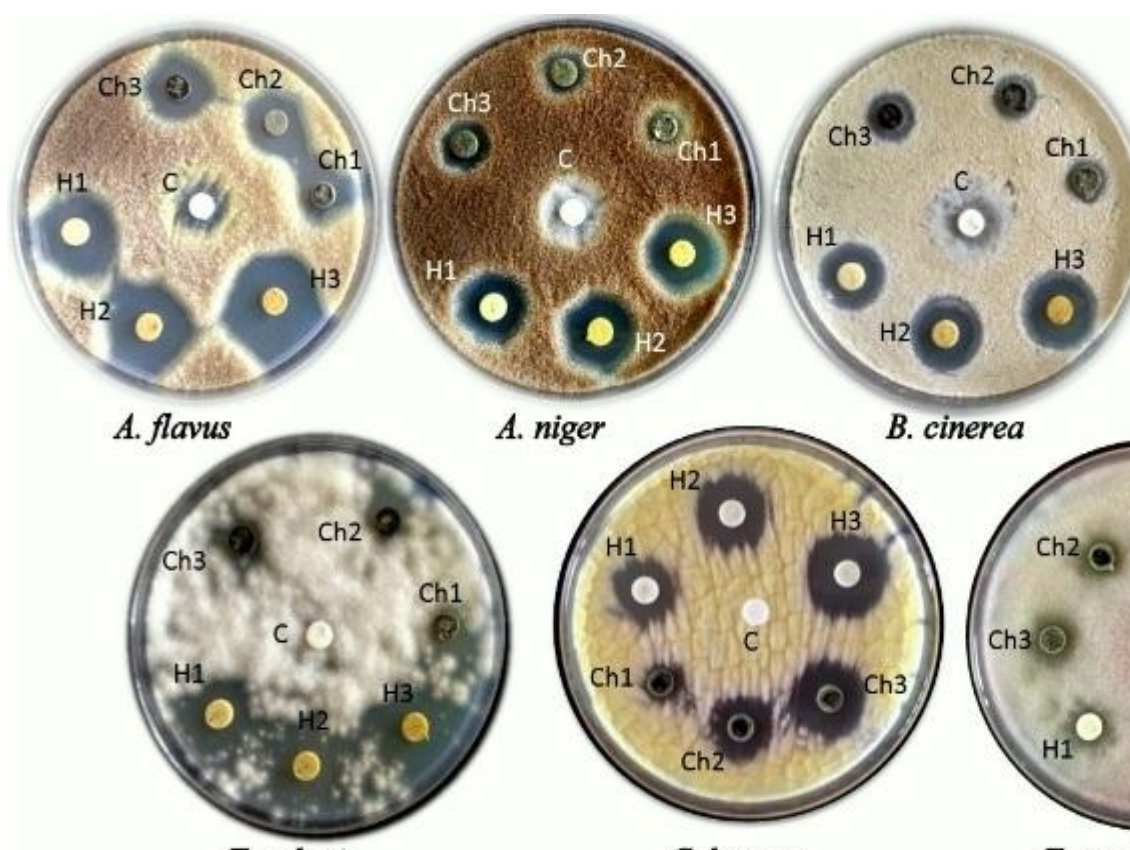

F. solani
A. niger

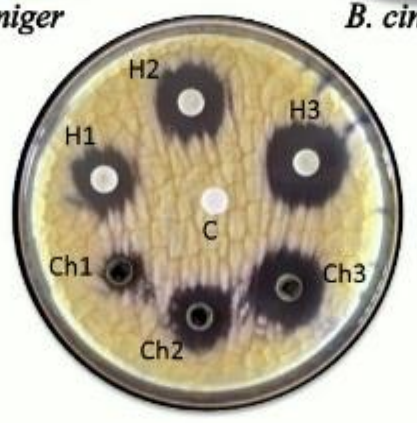

C. lunata

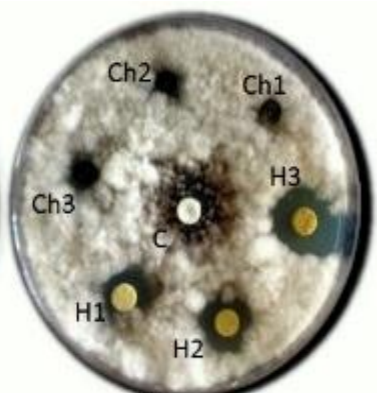

C. oryzea

Figure 1. Antifungal activity of hexane extract of $B$. mollis against phytopathogenic fungi

Hexane extract: H1- $1.25 \mathrm{mg}, \mathrm{H} 2-2.50 \mathrm{mg}, \mathrm{H} 3-5 \mathrm{mg}$. Chloroform: Ch1- $1.25 \mathrm{mg}$, Ch2- $2.50 \mathrm{mg}$, Ch2- 5mg. C - control (hexaconazol) $5 \mathrm{mg}$

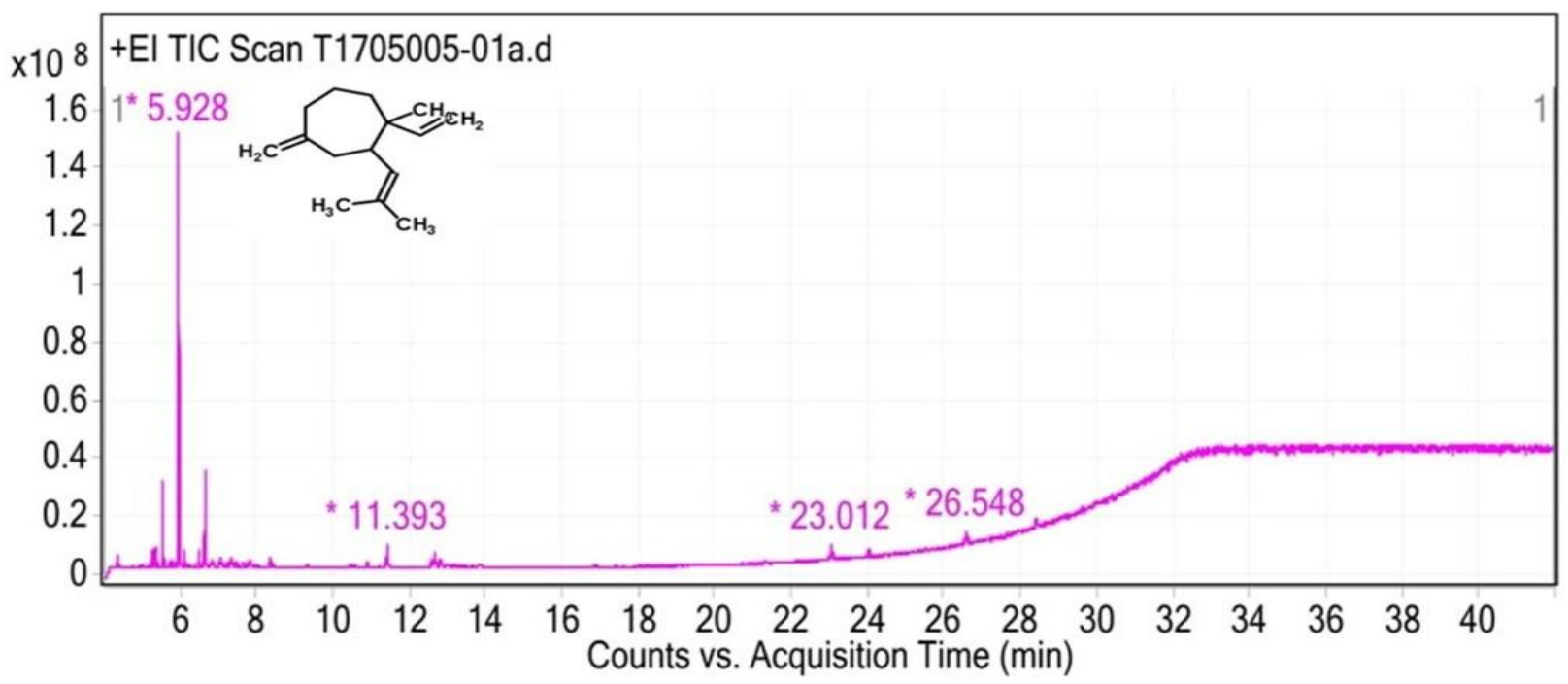

Figure 2) GCMS chromatogram of hexane extract of B. mollis showing the major compound 
Table 1: List showing the names, parts used, place and month of collection and voucher specimen number of different medicinal plants used in the antifungal screening experiment

\begin{tabular}{|c|c|c|c|c|c|}
\hline Botanical name and family & Local name & Collection place & $\begin{array}{l}\text { Collection stage } \\
\text { of plant and } \\
\text { month }\end{array}$ & $\begin{array}{l}\text { Plant } \\
\text { part } \\
\text { used }\end{array}$ & $\begin{array}{l}\text { voucher } \\
\text { specimen } \\
\text { number }\end{array}$ \\
\hline $\begin{array}{l}\text { Anisomeles malabarica (Linn). } \\
\text { Lamiaceae }\end{array}$ & Pei miratti & $\begin{array}{l}\text { Pitchavaram } \\
\text { mangrove forest }\end{array}$ & $\begin{array}{l}\text { Flowering } \\
\text { stage; May }\end{array}$ & $\begin{array}{l}\text { Whole } \\
\text { plant }\end{array}$ & $\overline{\text { ERISS-01 }}$ \\
\hline $\begin{array}{l}\text { Atlantia monophylla (Linn). } \\
\text { Rutaceae }\end{array}$ & Kattu elumichai & $\begin{array}{l}\text { Pitchavaram } \\
\text { mangrove forest }\end{array}$ & $\begin{array}{l}\text { Fruiting stage; } \\
\text { July }\end{array}$ & Leaf & ERISS-02 \\
\hline Blumea lacera (Linn). Asteraceae & Kaatumullangi & $\begin{array}{l}\text { Pitchavaram } \\
\text { mangrove forest }\end{array}$ & $\begin{array}{l}\text { Flowering } \\
\text { stage; June }\end{array}$ & Leaf & ERISS-03 \\
\hline $\begin{array}{l}\text { Blumea mollis (D.Don) Merr. } \\
\text { Asteraceae }\end{array}$ & Soft blumea & $\begin{array}{l}\text { Cuddaloredistric } \\
\text { (Akkravaram } \\
\text { lake forest) }\end{array}$ & $\begin{array}{l}\text { Flowering } \\
\text { stage; May }\end{array}$ & leaf & ERISS-04 \\
\hline $\begin{array}{l}\text { Clerodendrum inerme (Linn). } \\
\text { Lamiaceae }\end{array}$ & Sangamkuppi & $\begin{array}{l}\text { Loyola college } \\
\text { campus }\end{array}$ & $\begin{array}{l}\text { Flowering } \\
\text { stage; June }\end{array}$ & Leaf & ERISS-05 \\
\hline $\begin{array}{l}\text { Croton bonplandianum (Linn). } \\
\text { Euphorbiaceae }\end{array}$ & Rail poondu & $\begin{array}{l}\text { Cuddalore } \\
\text { district; } \\
\text { (Akkravaram } \\
\text { lake forest) }\end{array}$ & $\begin{array}{l}\text { Fruiting stage; } \\
\text { May }\end{array}$ & $\begin{array}{l}\text { Arial } \\
\text { part }\end{array}$ & ERISS-06 \\
\hline $\begin{array}{l}\text { Cordia dichotoma (Linn). } \\
\text { Boraginaceae }\end{array}$ & Kalvirusu & Avadi & $\begin{array}{l}\text { Fruiting stage; } \\
\text { June }\end{array}$ & Leaf & ERISS-07 \\
\hline $\begin{array}{l}\text { Murraya paniculata (Linn). } \\
\text { Rutaceae }\end{array}$ & Konjipazham & $\begin{array}{l}\text { Cuddalore } \\
\text { district } \\
\text { (Akkravaram } \\
\text { lake forest) }\end{array}$ & $\begin{array}{l}\text { Fruiting stage; } \\
\text { June }\end{array}$ & Leaf & ERISS-08 \\
\hline $\begin{array}{l}\text { Mollugo cerviana (Linn). } \\
\text { Molluginaceae }\end{array}$ & Parpadagum & $\begin{array}{l}\text { Cuddalore } \\
\text { district } \\
\text { (Akkravaram } \\
\text { lake forest) }\end{array}$ & Flowering; June & $\begin{array}{l}\text { whole } \\
\text { plant }\end{array}$ & ERISS-09 \\
\hline $\begin{array}{l}\text { Pedilanthus tithymaloides (Linn), } \\
\text { Euphorbiaceae }\end{array}$ & Kannaadikkallli & Avadi & $\begin{array}{l}\text { Dormant stage; } \\
\text { June }\end{array}$ & Leaf & ERISS-10 \\
\hline $\begin{array}{l}\text { Quisqualis indica (Linn) } \\
\text { Combretacea }\end{array}$ & Iranganmalli & Avadi & Flowering & Leaf & ERISS-11 \\
\hline Streblus asper (Lour) Moraceae & Paraimaram & $\begin{array}{l}\text { Loyola college } \\
\text { campus }\end{array}$ & $\begin{array}{l}\text { Flowering } \\
\text { stage; June }\end{array}$ & Leaf & ERISS-12 \\
\hline $\begin{array}{l}\text { Sphaeranthus indicus (Linn) } \\
\text { Asteraceae }\end{array}$ & $\begin{array}{l}\text { Karanthai/kotta } \\
\text { karanthai }\end{array}$ & $\begin{array}{l}\text { Cuddalore } \\
\text { district } \\
\text { (Akkravaram } \\
\text { lake forest) }\end{array}$ & $\begin{array}{l}\text { Flowering } \\
\text { stage; May }\end{array}$ & $\begin{array}{l}\text { Aerial } \\
\text { part }\end{array}$ & ERISS-13 \\
\hline Secamo neemetica (Retz) & Siruathankodi & Cuddalore & Dormant stage; & Aerial & \\
\hline
\end{tabular}


Asclepiadaceae

Tephrosia purpurea (Linn).

Fabaceae

Toddalia asiatica (Linn).

Rutaceae

Wedelia calendulacea (Linn). Asteraceae district

(Akkravaram

lake forest)

Pitchavaram mangrove forest

district

(Akkravaram

lake forest)

Cuddaloredistric

(Akkravaram lake forest)
June

part

ERISS-14

Dormant stage;

July

Whole

plant

Flowering

stage; June

Aerial

part

ERISS-16

Flowering stage; July

ERISS-15
Cuddalore

Ponnirachi

Table 2: Antifungal activity of hexane, chloroform and methanol extracts of 17 medicinal plant extracts against phytopathogenic fungi by disc diffusion method.

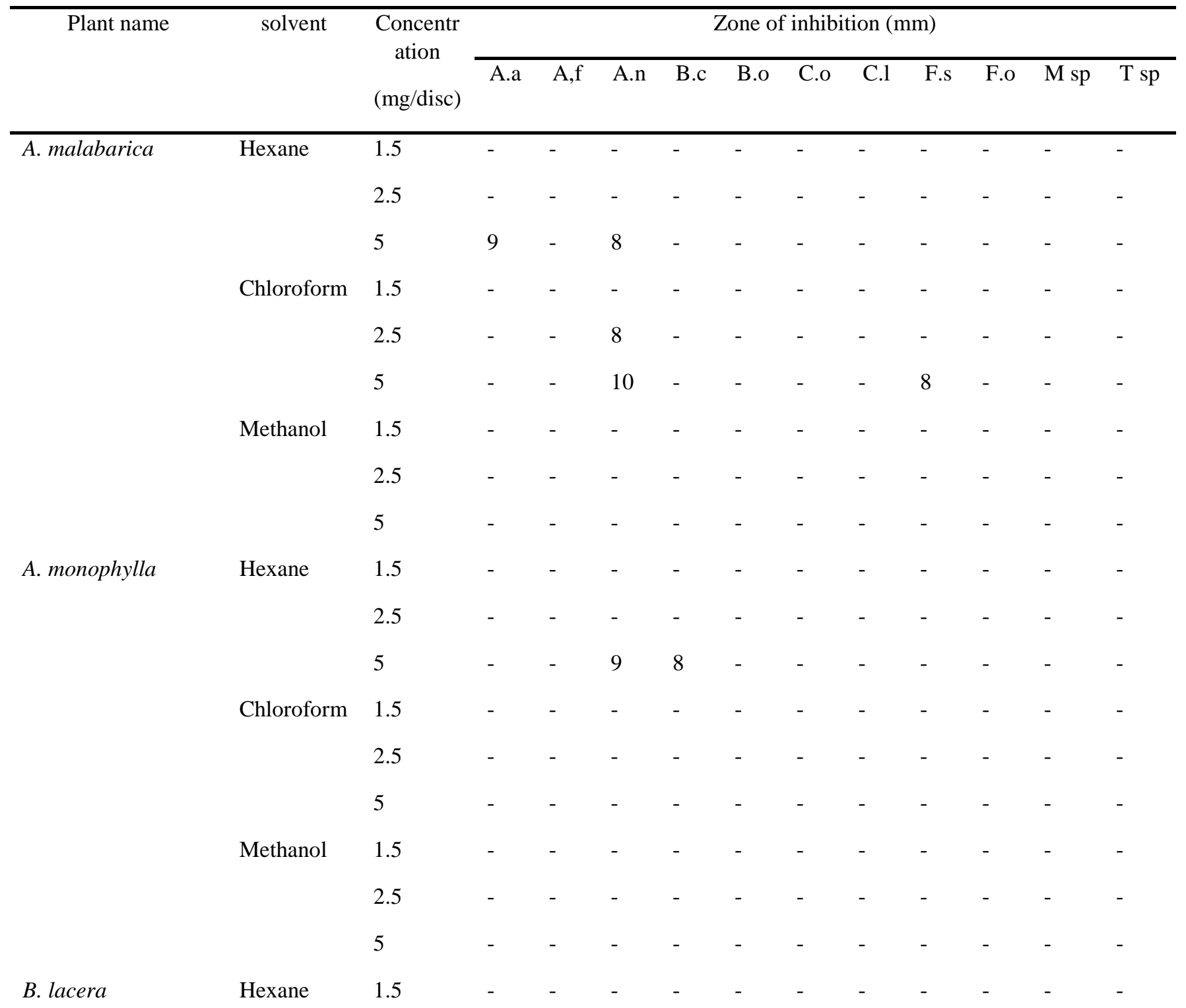




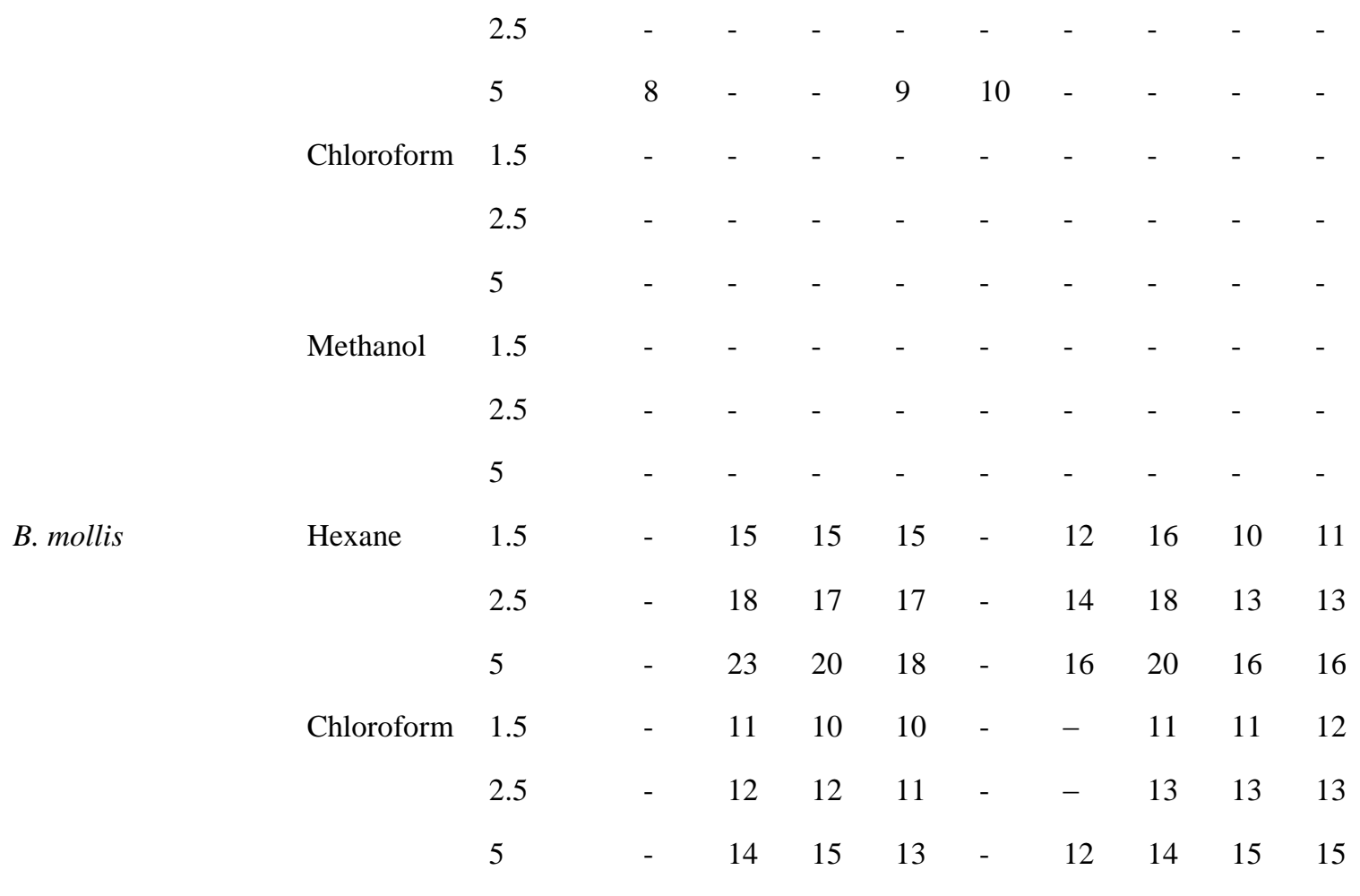

Methanol $\quad 1.5$

5

C. inerme

Hexane $\quad 1.5$

5

Chloroform $\quad 1.5$

2.5

5

Methanol $\quad 1.5$

$$
2.5
$$

5

C. bonplandianum Hexane $\quad 1.5$

2.5

Chloroform 1.5 


\section{5}

Methanol $\quad 1.5$

$$
2.5
$$

5

C. dichotoma

Hexane $\quad 1.5$

5

Chloroform $\quad 1.5$

5

Methanol $\quad 1.5$

5

M. paniculata Hexane $\quad 1.5$

$$
2.5
$$

5

8

8

Chloroform $\quad 1.5$

$$
2.5
$$

$$
5
$$

Methanol $\quad 1.5$

5

M. cerviana

Hexane $\quad 1.5$

5

Chloroform $\quad 1.5$

5

Methanol $\quad 1.5$

5 
P. tithymaloides Hexane $\quad 1.5$

5

Chloroform $\quad 1.5$

2.5

5

Methanol $\quad 1.25$

2.5

5

Q. indica

Hexane $\quad 1.5$

2.5

5

Chloroform $\quad 1.5$

2.5

5

Methanol $\quad 1.5$

2.5

5

S. asper

Hexane $\quad 1.5$

5

Chloroform $\quad 1.5$

2.5

5

Methanol $\quad 1.5$

2.5

5

S. indicus

$\begin{array}{lll}\text { Hexane } & 1.5 & - \\ 2.5 & 8 \\ 5 & 9\end{array}$

Chloroform $\quad 1.5$ 
2.5

5

Methanol $\quad 1.5$

5

S. emetica

Hexane $\quad 1.5$

2.5

5

Chloroform $\quad 1.5$

2.5

5

Methanol $\quad 1.5$

2.5

5

T. purpurea

Hexane $\quad 1.5$

5

9

$8 \quad 8$

$-9$

.5

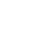

.5

.5

.5

5

\section{T. purpurea}

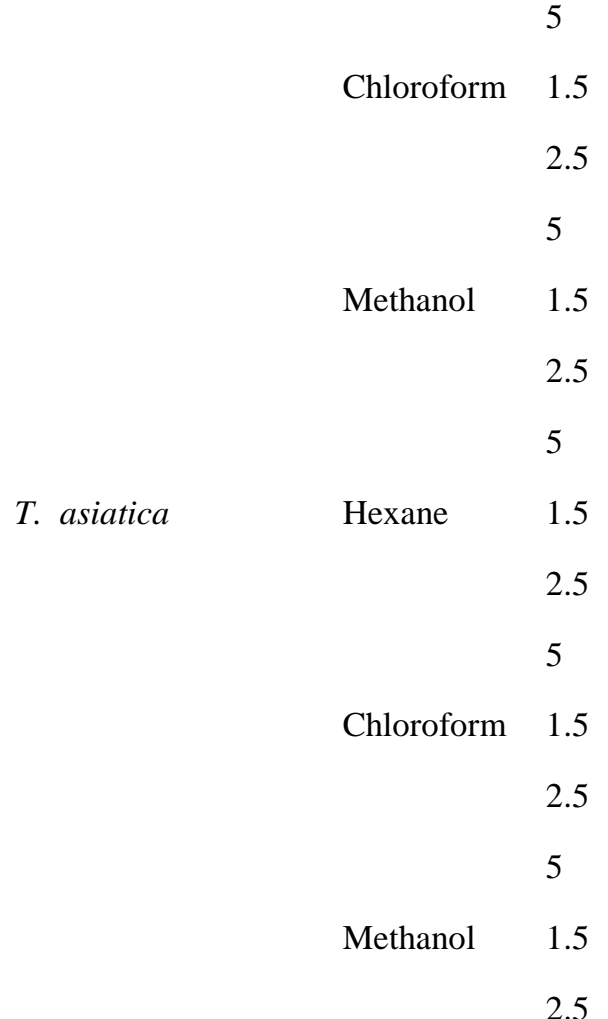




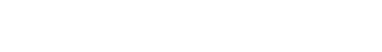

5

W. calendulacea Hexane $\quad 1.5$

$\begin{array}{cccccc}2.5 & - & - & - & - & - \\ 5 & & - & - & - & -\end{array}$

Chloroform 1.5

2.5
5

Methanol $\quad 1.5$

$$
5
$$

Standared control (Hexaconazole) 5

$11 \quad 12 \quad 10$

15

$12 \quad 15$

A.a.-A. alternata, A.f-A. flavus, A.n-A. niger, B.c-B. cineriae, B.o-B. oryzea, C.o- C. oryzea, C.l- C. lunata, F.s-F. solani, F.o- F. oxysporum, - no activity.

Table 3: Antifungal activity of hexane and chloroform extracts of $B$. mollis by disc diffusion method

\begin{tabular}{|c|c|c|c|c|c|c|c|c|}
\hline \multirow[t]{2}{*}{ Extract } & \multirow{2}{*}{$\begin{array}{l}\text { Conc. }(\mathrm{mg} / \\
\text { disc })\end{array}$} & \multicolumn{7}{|c|}{ Zone of inhibition (mm) } \\
\hline & & $\overline{A, f}$ & A.n & B.c & C.o & C.1 & F.s & F.o \\
\hline \multirow[t]{3}{*}{ Hexane } & 1.5 & $15.33 \pm 0.77$ & $15 \pm 0$ & $15.33 \pm 0.57$ & $12 \pm 1$ & $16.33 \pm 0.57$ & $14 \pm 0$ & $11.33 \pm 0.57$ \\
\hline & 2.5 & $18.33 \pm 0.57$ & $16.66 \pm 0.57$ & $17 \pm 0$ & $13.66 \pm 0.57$ & $17.66 \pm 0.57$ & $15.33 \pm 0.57$ & $12.66 \pm 0.57$ \\
\hline & 5 & $23 \pm 1$ & $18.33 \pm 0.57$ & $18.33 \pm 0.57$ & $16.33 \pm 0.57$ & $20.66 \pm 0.57$ & $17.33 \pm 0.57$ & $14.33 \pm 0.57$ \\
\hline \multirow[t]{3}{*}{ Chloroform } & 1.5 & $11.66 \pm 1.15$ & $10.33 \pm 0.57$ & $10.66 \pm 0.57$ & - & $12.33 \pm 0.57$ & $11.33 \pm 0.57$ & $11.66 \pm 0.57$ \\
\hline & 2.5 & $12 \pm 0$ & $12.33 \pm 0.57$ & $11.66 \pm 0.57$ & - & $14.33 \pm 0.57$ & $13.33 \pm 0.57$ & $12.33 \pm 0.57$ \\
\hline & 5 & $13.66 \pm 0.57$ & $14.66 \pm 0.57$ & $13 \pm 0$ & $11.66 \pm 0.7$ & $15 \pm 1$ & $14.66 \pm 0.57$ & $14 \pm 0$ \\
\hline $\begin{array}{l}\text { Reference Control } \\
\text { (Hexaconazole) }\end{array}$ & 5 & $11 \pm 1$ & $12 \pm 0$ & $10 \pm 1$ & $11 \pm 0$ & - & - & - \\
\hline
\end{tabular}

A.f-A. flavus, A.n- A. niger, B.c-B. cineriae, C.o- C. oryzea, C.1- C. lunata, F.s- F. solani, F.o - F. oxysporum, Macrophomiana sp, Trichoderma sp, - no activity, Values are mean \pm standard error.

Table 4: MIC values (Mean \pm SE) of heaxane and chloroform extracts of $B$. mollis against phytopathogenic fungi.

\begin{tabular}{llll}
\hline \multirow{2}{*}{ Microorganism } & \multicolumn{3}{l}{ Minimal inhibitory concentration } \\
\cline { 2 - 4 } & Hexane extract $\mathrm{mg} / \mathrm{disc}$ & Hexaconazole* $(\mathrm{mg} / \mathrm{ml})$ & Carbendazim * $(\mathrm{mg} / \mathrm{ml})$ \\
\hline A. flavus & $0.625 \pm 1$ & $1.25 \pm 0$ & $1.25 \pm 0$ \\
A. niger & $0.625 \pm 0$ & $0.625 \pm 0$ & $0.625 \pm 0$ \\
B. cinearae & $0.625 \pm 2$ & $1.25 \pm 0$ & $1.25 \pm 0$ \\
C. oryzea & $1.25 \pm 1$ & - & $1.25 \pm 0$ \\
C. lunata & $0.3125 \pm 0$ & $5 \pm 0$ & $0.625 \pm 0$ \\
F. solani & $1.25 \pm 2$ & - & $0.312 \pm 0$ \\
F. oxysporum & $>5 \pm 1$ & - & $0.625 \pm 0$ \\
\hline
\end{tabular}

- No activity; *commercial fungicidal compounds, 
Table 5: Preliminary phytochemical analysis for B. mollis extracts

\begin{tabular}{llll}
\hline Test & Hexane & Chloroform & Methanol \\
\hline Tannins & - & - & + \\
Flavonoids & - & + & - \\
Terpenoids & + & + & + \\
Saponins & + & - & + \\
Steroids & - & + & + \\
Carbohydrates & - & + & + \\
Glycosides & & - & - \\
Coumarins & - & + & + \\
Alkaloids & - & + & - \\
Proteins & - & + & + \\
Anthraquinones & + & - & + \\
Anthocyanins & + & & \\
\hline
\end{tabular}

+ present; - absent

Table 6: GCMS analysis of hexane extract of B. mollis

\begin{tabular}{|c|c|c|c|}
\hline Peak no & compound name & $\begin{array}{l}\text { Retention } \\
\text { time }\end{array}$ & $\begin{array}{l}\text { Area of } \\
\text { percentage }\end{array}$ \\
\hline 1. & 6-Tridecene, $(\mathrm{Z})$ - & 4.332 & 2.29 \\
\hline 2. & 6-Tridecene, $(\mathrm{Z})$ - & 5.228 & 3.61 \\
\hline 3. & beta.-curcumene & 5.265 & 2.85 \\
\hline 4. & $\begin{array}{l}\text { Cyclohexane, 1-ethenyl-1-methyl-2,4-bis(1-methylethenyl)-, [1S- } \\
\text { (1.alpha.,2.beta.,4.beta.)]- }\end{array}$ & 5.326 & 4.19 \\
\hline 5. & 4-tert-Butylcatechol, dimethyl ether & 5.409 & 1.15 \\
\hline 6. & Isocaryophyllene & 5.529 & $18.24^{*}$ \\
\hline 7. & 7-Pentadecen-5-yne, (Z)- & 5.725 & 1.82 \\
\hline 8. & 8,11-Octadecadiynoic acid, methyl ester & 5.824 & 1.92 \\
\hline 9. & $\begin{array}{l}\text { Cycloheptane, 4-methylene-1-methyl-2-(2-methyl-1-propen-1-yl)-1- } \\
\text { vinyl }\end{array}$ & 5.928 & $34.21^{*}$ \\
\hline 10. & $\begin{array}{l}\text { Azulene, 1,2,3,3a,4,5,6,7-octahydro-1,4-dimethyl-7-(1-methylethenyl)- } \\
\text {, [1R-(1.alpha.,3a.beta.,4.alpha.,7.beta.)]- }\end{array}$ & 5.976 & 4.24 \\
\hline 11. & 4-epi-cubedol & 6.088 & 5.44 \\
\hline 12. & 6-Tridecene, $(\mathrm{Z})$ - & 6.47 & 4.04 \\
\hline 13. & Caryophyllene oxide & 6.626 & $26.73 *$ \\
\hline 14. & Caryophyllene oxide & 6.827 & 2.61 \\
\hline 15. & 5,8,11,14-Eicosatetraenoic acid, methyl ester, (all-Z)- & 7.028 & 5.15 \\
\hline 16. & cis-5,8,11,14,17-Eicosapentaenoic acid & 7.294 & 2.36 \\
\hline 17. & 5,8,11,14-Eicosatetraenoic acid, methyl ester, (all-Z)- & 7.391 & 1.49 \\
\hline 18. & 5,8,11,14-Eicosatetraenoic acid, methyl ester, (all-Z)- & 7.456 & 1.06 \\
\hline 19. & 8,11-Octadecadiynoic acid, methyl ester & 7.675 & 1.56 \\
\hline 20. & cis-Z-.alpha.-Bisabolene epoxide & 7.802 & 3.32 \\
\hline 21. & 1-Eicosano & 8.336 & 2.88 \\
\hline 22. & Butyrylthioacetic acid, ethyl ester & 11.393 & 9.6 \\
\hline 23. & Phytol & 12.575 & 4.03 \\
\hline 24. & Tetraacetyl-d-xylonic nitrile & 12.635 & 7.06 \\
\hline 25. & Bicyclo[2.2.1]heptan-2-one, 4,7,7-trimethyl-, semicarbazone & 12.785 & 4.6 \\
\hline 26. & 2,4,6-Cycloheptatrien-1-one, 3,5-bis-trimethylsilyl & 23.012 & 8.34 \\
\hline 27. & Hexasiloxane, $1,1,3,3,5,5,7,7,9,9,11,11$-dodecamethyl & 26.548 & 4.81 \\
\hline
\end{tabular}

\footnotetext{
*-major compound
} 
Acknowledgments - The authors are thankful to Entomology Research Institute, Loyola College, Chennai for financial assistance and facilities.

Author Contributions S. Sivanandhan did the experiment; A. Jackson, P. Ganesan, Sylvester Darvin and M. Gabriel Paulraj helped in the collection of the data, S. Ignacimuthu designed the outline of the experiment and oversaw the work. All authors contributed in manuscript preparation.

Conflicts of Interest- The authors declare that there are no conflicts of interest

\section{REFERENCES}

[1]. D. Prusky. Reduction of the incidence of postharvest quality losses, and future prospects. Food Security 3: 463-474, 2011

[2]. S. Savary, A. Ficke, J.N. Aubertot, C. Hollier. Crop losses due to diseases and their implications for global food production losses and food security. Food Security 4: 519-537, 2012

[3]. M.Y.Yoon. B. Cha, J.C. Kim. Recent trends in studies on botanical fungicides in agriculture. Plant Pathol. J 29: 1-9, 2013

[4]. C.A. Breda, A.M. Gasperini, V.L. Garcia, K.M. Monteiro, G.A. Bataglion, M.N. Eberlin, M.C.T. Duarte. Phytochemical analysis and antifungal activity of extracts from leaves and fruit residues of brazilian savanna plants aiming its use as safe fungicides. Nat Prod Bioprospect 6: 195-204, 2016

[5]. S. Singh, S. Kaur, D. Singh. Toxicological profile of Indian foods-ensuring food safety in India. Food Safety in the 21st Century: Public Health Perspective, Chapter 9,111, 2016

[6]. G. Matthews. Pesticides: health, safety and the environment. $2^{\text {nd }}$ edn. John Wiley \& Sons, UK. 2015

[7]. S. Rastegar, M. Gozari. Effect of mangrove plant extract on growth of four fungal pathogens. J Paramed Sci 8, 2016

[8]. L.R. Goldman, S. Koduru. Chemicals in the environment and developmental toxicity to children: a public health and policy perspective. Environmental health perspectives. 108 (Suppl 3): 443, 2000

[9]. P. Fernando, Carvalho. Agriculture, pesticides, food security and food safety, Environ Sci Policy 9, 7-8, 685-692, 2006

[10]. E.M. Soylu, Ş. Kurt. S. Soylu. In vitro and in vivo antifungal activities of the essential oils of various plants against tomato grey mould disease agent Botrytis cinerea. Int. J. Food Microbiol 143: 183-189, 2010.

[11]. C.M. Abraham, S. Prasad. Preliminary phytochemical screening and antifungal activity of bilimbi and papaya leaf extract on fungal pathogens, Int. j. sci. res. biol. Sci, 5, 4, 97-102, 2018

[12]. F. Bourgaud, A Gravot, S. Milesi, E Gontier. Production of plant secondary metabolites: a historical perspective. Plant science. 1; 161: 839-51. 2001

[13]. A.E Edris. Pharmaceutical and therapeutic potentials of essential oils and their individual volatile constituents: a review. Phytotherapy Research: An International Journal Devoted to Pharmacological and Toxicological Evaluation of Natural Product Derivatives. 21, 4: 30823. 2007

[14]. J.L. Rios, M.C. Recio. Medicinal plants and antimicrobial activity. Journal of ethnopharmacology. 100, (1-2): 80-4, 2005.

[15]. S.I. Kim, J.Y. Roh, D.H. Kim, H.S. Lee, Y.J. Ahn. Insecticidal activities of aromatic plant extracts and essential oils against Sitophilus oryzae and Callosobruchus chinensis. Journal of Stored products research. 39, 3: 293-303. 2003

[16]. I.K. Park, J. Kim, S.G. Lee, S.C. Shin. Nematicidal activity of plant essential oils and components from ajowan (Trachyspermum ammi), allspice (Pimenta dioica) and litsea (Litsea cubeba) essential oils against pine wood nematode (Bursaphelenchus xylophilus). Journal of nematology. 39, 3:275, 2007

[17]. S.M. Rates. Plants as source of drugs. Toxicon. 39, $5: 603-13,2001$

[18]. S. Adikay, A. Aneesha. In vitro antioxidant studies of ethanolic extract of leaves of Blumea mollis. Int. J. Pharma. Bio. Sci 3: 764-769, 2012

[19]. D.N. Guha Bakshi, P. Sensarma, D.C. Pal. Lexicon of medicinal plants in India. Calcutta, India : Naya Prokash Publisher, 1999

[20]. V.K. Ratnam, V.R. Raju. Folk medicine used for common women ailments adivasis in the eastern Ghats of Andhra Pradesh, Indian Journal of Treaditianal Knowledge 4, 267-270, 2005

[21]. F. Mauch, B. Mauch-Mani. T. Boller. Antifungal hydrolases in pea tissue II. Inhibition of fungal growth by combinations of chitinase and ß-1, 3-glucanase. Plant Physiol, 88: 936-942, 1988

[22]. J. Hudzicki. Kirby-Bauer disk diffusion susceptibility test protocol. ASM Microbe Library, American Society for Microbiology. 2009

[23]. P.A. Wayne. Clinical and Laboratory Standards Institute Reference method for broth dilution antifungal susceptibility testing of filamentous fungi; approved standard CLSI document M38-A2 Clinical and Laboratory Standards Institute, 2008.

[24]. M. Yadav, S. Chatterji, S.K. Gupta, G. Watal. Preliminary phytochemical screening of six medicinal plants used in traditional medicine. Int J Pharm Pharm Sci 6: 539-542, 2014

[25]. D.S. Fabricant, N.R. Farnsworth. The value of plants used in traditional medicine for drug discovery. Environ Health Perspect 109: 69, 2001

[26]. M.D.C. Sales, H.B. Costa, P.M.B. Fernandes, J.A. Ventura, D.D. Meira. Antifungal activity of plant extracts with potential to control plant pathogens in pineapple. Asian Pac J Trop Biomed 6: 26-31, 2016.

[27]. S. Adikay, A. Aneesha. In vitro antioxidant studies of ethanolic extract of leaves of Blumea mollis. Int. J. Pharma. Bio. Sci 3: 764-769, 2012

[28]. G. Devi, K. Revathi. Evaluation of hepatoprotective activity of Blumea mollis D. Don Merr. on paracetamol-induced hepatotoxicity in rats. Research Journal of Pharmacology and Pharmacodynamics 4: 206-209, 2012 
[29]. M. Chandrasekaran. V. Venkatesalu. Antibacterial and antifungal activity of Syzygium jambolanum seeds. J. Ethnopharmacol 91: 105-108, 2004

[30]. P. Alade, O. Irobi. Antimicrobial activities of crude leaf extracts of Acalypha wilkesiana. J. Ethnopharmacol 39: 171-174, 1993

[31]. M. Parimala, F.G. Shoba. In vitro antimicrobial activity and HPTLC analysis of hydroalcoholic seed extract of Nymphaea nouchali Burm. F. BMC Complement Altern Med 14: 361, 2014

[32]. B. Mahesh, S. Satish. Antimicrobial activity of some important medicinal plant against plant and human pathogens. World journal of agricultural sciences 4: 839-843, 2008

[33]. D.J. de Rodríguez. F.A. Trejo-González, R. Rodríguez-García, M.L.V. Díaz-Jimenez, A. Sáenz-Galindo, F.D. Hernández-Castillo, J.A. Villarreal-Quintanilla, and F.M. Peña-Ramos. Antifungal activity in vitro of Rhus muelleri against Fusarium oxysporum $\mathrm{f}$. Sp. Lycopersici. Ind Crops Prod 75: 50-158, 2015

[34]. A. Senthilkumar, K. Kannathasan, V. Venkatesalu. Chemical constituents and larvicidal property of the essential oil of Blumea mollis (D. Don) Merr. against Culex quinquefasciatus. Parasitology research 1034: 959-962, 2008

[35]. D. Yang. L. Michel, J.P. Chaumont, J. Millet-Clerc. Use of caryophyllene oxide as an antifungal agent in an in vitro experimental model of onychomycosis. Mycopathologia 2: 79-82, 2000

[36]. D.Yang, L. Michel, J.P. Chaumont, J. Millet-Clerc.Use of caryophyllene oxide as an antifungal agent in an in vitro experimental model of onychomycosis. Mycopathologia. 1;148:79-82, 2000 